\title{
Simple Phenomenological Model for Phase Transitions in \\ Confined Geometry. 2: Capillary Condensation/Evaporation in Cylindrical Mesopores
}

Roland J. -M. Pellenq ${ }^{1 *}$, Benoit Coasne ${ }^{2}$, Renaud O. Denoyel ${ }^{3}$, O. Coussy ${ }^{4}$

${ }^{1}$ Centre Interdisciplinaire des Nanosciences de Marseille, CINaM, UPR CNRS 3118, Campus de Luminy, 13288 Marseille Cedex 09, France and Department of Civil and Environmental Engineering, Massachusetts Institute of Technology, 77 Massachusetts Avenue, Cambridge, 02139 MA, USA.

${ }^{2}$ Institut Charles Gerhardt Montpellier, UMR CNRS 5253, Université Montpellier, Place Eugène Bataillon, 34095 Montpellier Cedex 05, France.

${ }^{3}$ Laboratoire Chimie Provence, UMR CNRS 6121, Université de Provence, Centre de Jérôme, 13397 Marseille Cedex 09, France.

${ }^{4}$ Université de Paris-Est, Institut Navier, Ecole Nationale des Ponts et Chaussées - 6-8 Av. Blaise Pascal - Cité Descartes F 77455, Marne-la-Vallée Cedex 2, France,

Version Monday, August 07, 2017

* To whom correspondence should be sent. pellenq@cinam.univ-mrs.fr 
Abstract. A simple phenomenological model that describes capillary condensation and evaporation of pure fluids confined in cylindrical mesopores is presented. Following the work of Celestini (Phys. Lett. A, 1997, 228, 84), the free energy density of the system is derived using interfacial tensions and a corrective term that accounts for the interaction coupling between the vapor/adsorbed liquid and the adsorbed liquid/adsorbent interfaces. This corrective term is shown to be consistent with the Gibbs adsorption isotherm and assessed by standard adsorption tests. This model reveals that capillary condensation and evaporation are metastable and equilibrium processes respectively, hence exhibiting the existence of a hysteresis loop in adsorption/desorption isotherms that is well known in experiment. We extend the phenomenological model of Celestini to give a quantitative description of adsorption on the pore wall and hysteresis width evolution with temperature and confinement. Direct quantitative comparison is made with experimental data for confined argon. Used as a characterizing tool, this integrated model allows in a single fit of an experimental adsorption/desorption isotherm assessing essential characterization data such as the specific surface area, pore volume, and mean pore size. 


\section{Introduction}

Nanoporous materials are made up of pores having a size of a few nanometers that are either unconnected/independent or connected. ${ }^{1,2}$ Porous materials with independent pores include solids with either crystalline (porous silicon, ${ }^{3}$ carbon nanotubes ${ }^{4}$ ) or amorphous pore walls $\left(\mathrm{MCM}-41^{5}, \mathrm{SBA}-15^{6}\right)$. Networked porous solids are usually glasses such as $\mathrm{Vycor}^{7}$ or $\mathrm{CPG}^{8}$. Condensation and/or freezing of molecular fluids within nanoporous materials are routinely used for characterization purpose using macroscopic physical laws such as the well know Kelvin or Gibbs-Thompson equations that link a thermodynamic quantity to the mean pore size. ${ }^{9,10}$ These approaches implicitly assume that such phenomena are first order transitions involving phase coexistence between the gas and liquid or the solid and liquid phases. The stability of the confined adsorbate is described using gas/liquid or liquid/solid surface tensions. Capillary condensation in nanoporous materials is usually an irreversible phenomenon, as revealed by significant and reproducible hysteresis loops observed during adsorption/desorption cycles (amount adsorbed versus pressure). ${ }^{1}$ Such a hysteresis loop shrinks and disappears as the temperature increases. ${ }^{11,12,13,14,15}$ This hysteretic behavior, which is common to both nanoporous materials with independent, unconnected pores or connected pores, is often explained using one of the following model. ${ }^{16}$ In the case of connected pores, the hysteresis loop is ascribed to the existence of constrictions or bottlenecks between pores that cause a delay in the desorption process compared to the adsorption ${ }^{17,18,19}$. In contrast, hysteresis loops observed for regular unconnected pores are usually interpreted as a change in the symmetry of the gas/liquid interface upon filling and emptying mechanisms ${ }^{16,20,21}$. Density Functional or Lattice Gas theories have shown that the first order character of fluid condensation is relevant for unconnected slit pores only ${ }^{22}$. Indeed, fluids in very narrow cylindrical pores are one dimensional systems and, therefore, cannot exhibit stricto sensus a first order transition. ${ }^{22,23}$ On the other hand, one expects fluids confined in cylindrical 
mesopores large enough to behave as a quasi three-dimensional system. In other words, there must be a minimum pore size for which the confined fluid recovers its bulk properties. In the case of disordered and/or connected porous materials, recent works have shown that capillary condensation cannot be considered as a first order transition; the morphological and topological disorders generate a complex free energy landscape with many metastable states that prevents the system from following the "at equilibrium" thermodynamic path ${ }^{24,25,26}$. However, the picture remains unclear since these results were obtained for very large confined molecules in mesopores (i.e. using very large lattice spacing); the same calculation repeated for "normal" size molecules confined into a big chunk of Vycor, showed the same temperature dependence of the hysteresis loop width as observed for large enough cylindrical and slit pores. ${ }^{27}$ As in the case of simple pore geometry, one expects fluids confined in disordered porous materials with pores large enough would behave as a quasi threedimensional system. Recent lattice gas calculations by Detcheverry et al. $^{28}$ supports this intuition; the authors found evidence of a first order transition in the case of an aerogel sample with a porosity of $95 \%$, while such a transition was not observed for a similar porous structure with a porosity of $87 \%$.

In this work, we report a simple phenomenological model based on a generalized Gibbs surface dividing theory ${ }^{29}$ for adsorption and capillary condensation/evaporation in cylindrical mesopores (the slit pore geometry will be considered in a separate paper) ${ }^{30}$. The model, which does not require a detailed description of the system at the molecular level, is derived following the same approach as that reported in our previous work for the liquid/solid transition in confined geometry. ${ }^{31}$ It is shown that the present model describes well the main features of gas/liquid transitions of simple molecular fluids confined in independent and unconnected cylindrical mesopores. The remainder of the paper is organized as follows. We first present the model and underlying concepts. Then, we give the different steps to derive 
necessary model parameters and compare calculated adsorption/desorption isotherms to experiment in the case of Ar confined in MCM-41 nanoporous materials of various pore dimensions and at several temperatures.

\section{Model for the interactions between interfaces}

Let us consider a system composed of three phases 1,2 , and 3 separated by two interfaces $1 / 2$ and 2/3 (see Figure 1). These interfaces are characterized at the microscopic scale by gradients of the physical properties of the system, such as the density of particles for instance. Thermodynamics, which deals with homogeneous phases only, cannot be used to describe such inhomogeneous systems. However, it is possible to overcome this problem by using the Gibbs surface dividing theory ${ }^{29}$ in which the system shown in Figure 1 is modelled as three homogeneous phases separated by infinitely thin, fictive interfaces that are located in the real interfacial regions from the concept of surface excess adsorbed amount defined as the excess of the amount of adsorbed molecules actually present in the interfacial layer over that which would be present at the same equilibrium gas pressure in a bulk reference system. In this interfacial adsorbed layer, the gas phase concentration is constant up to the Gibbs surface, and the reference concentration of the adsorbing fluid is zero beyond it, up to the solid surface. Obviously such a formal definition is becoming unclear in the case of a layer made of a few molecular size thicknesses. 


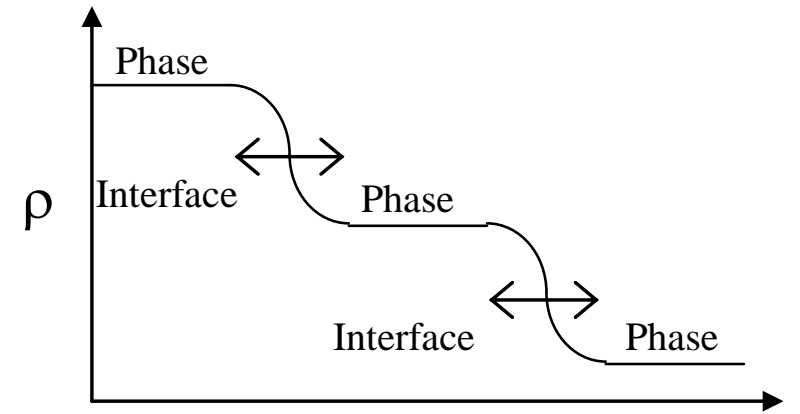

Z

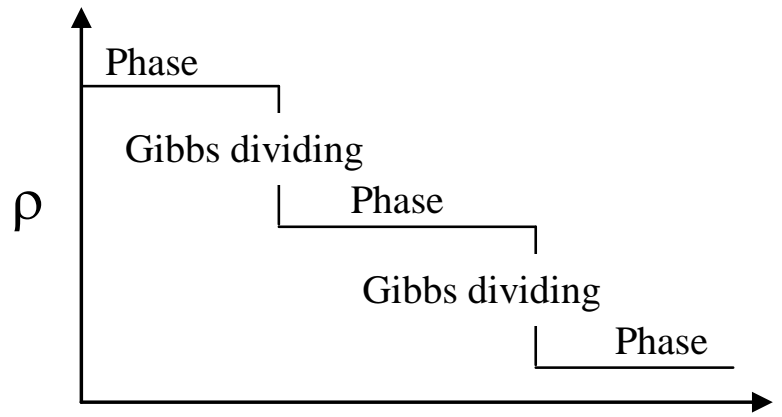

$\mathrm{Z}$

Figure 1. (left) A real system is composed of three phases 1, 2, and 3 separated by two interfaces $1 / 2$ and 2/3. (Right) Using the Gibbs surface dividing theory, the system is modelled as three homogeneous phases separated by infinitely thin, fictive interfaces that are located in the middle of the real interfaces.

Within the frame of the Gibbs surface dividing theory, the grand free energy of such a system can be expressed as:

$$
\Omega=-P_{1} V_{1}-P_{2} V_{2}-P_{3} V_{3}+\gamma_{12} A_{12}+\gamma_{23} A_{23}
$$

where $V_{I}$ and $P_{I}$ are the volume and pressure of each phase, while $A_{I J}$ and $\gamma_{I J}$ are the surface area and surface tension of each interface $(I, J=1,2$ or 3$)$. In the case of macroscopic phases, interfaces $1 / 2$ and $2 / 3$ can be considered as independent as they are located far from each other. On the other hand, such an assumption breaks down for nanoscopic phases since the two interfaces are coupled because of the small separation distance between them, which becomes comparable with the characteristic length $\xi$ scaling the correlations between the two interfaces; i.e. variation of one interface induces a response of the other interface. Following the previous work by Celestini, ${ }^{32,33}$ equation (1) is rewritten in order to account for such an interaction by adding a coupling term $\varepsilon(t)$ :

$$
\Omega=-P_{1} V_{1}-P_{2} V_{2}-P_{3} V_{3}+\gamma_{12} A+\gamma_{23} A+\varepsilon(t) A
$$

Where $t$ is the distance between the two plane interfaces $1 / 2$ and $2 / 3$. Equation (2) assumes that the two interfaces have the same area, $A$. The coupling term must verify the following 
condition: when $t$ is much larger than the characteristic distance $\xi$ the correlations between the interfaces vanish. As a result the energy related to the interface reduces to $\gamma_{12} A+\gamma_{23} A$ so that $\varepsilon(t)$ tends to 0 . In contrast, when $t$ becomes zero, phase 2 disappears and the energy related to the interface reduces to $\gamma_{13} A$ so that $\varepsilon(t)$ tends to the spreading coefficient $S$ defined by:

$$
S=\left(\gamma_{13}-\gamma_{12}-\gamma_{23}\right) \varepsilon(t)
$$

These conditions requires the coupling term $\varepsilon(t)$ to have the following form: $:^{32,33,34}$

$$
\lim _{t \rightarrow 0} \varepsilon(t)=1 \quad \text { and } \quad \lim _{t \rightarrow \infty} \varepsilon(t)=0
$$

Among others possible functions, Celestini ${ }^{32,33}$ suggested the following $\varepsilon(t)$ function:

$$
\varepsilon(t)=\exp \left(\frac{-t}{\xi(T)}\right)
$$

As shown below, we introduce a dependency upon the temperature $T$ of the characteristic length $\xi$ scaling the interactions in the system. Equation (5) can be seen as a general solution that can be parameterized to reproduce experimental data.

\section{Adsorption on an infinite plane substrate}

Up to now the phases 1, 2 and 3 forming the system were unspecified. Consider now the case where the phase $1=V$ is a vapor phase, the phase $2=L$ the corresponding liquid phase adsorbed in the form of a thin layer of varying thickness $t$ onto a solid substrate forming the phase $3=S$. The existence of an adsorbed film at the solid substrate implies that the spreading coefficient $S=\gamma_{V S}-\gamma_{V L}-\gamma_{L S}$ has to be positive. ${ }^{34}$ In the following, the volume of the solid substrate will remain constant, while the gas occupies a volume of infinite extent. Accordingly considering only the change $\Delta \Omega$ in the grand free energy $\Omega$ and similarly to (2) one writes: 


$$
\Delta \Omega / A=-t\left(P_{L}-P_{V}\right)+\gamma_{L S}+\gamma_{L V}+\varepsilon(t)
$$

The adsorbed liquid and its vapor being in thermodynamic equilibrium, their pressures have to satisfy the Kelvin equation:

$$
P_{V}-P_{L}=-\rho_{L} \ln \left(\frac{P_{V}}{P_{0}}\right)
$$

where the liquid density $\rho_{L}$ has been assumed to be much larger than the vapor density $\rho_{V}{ }^{21,35}$ and $P_{0}$ is the vapor saturating pressure. Substitution of (7) in (6) leads to:

$$
\Delta \Omega / A=-t \rho_{L} k T \ln \left(\frac{P_{V}}{P_{0}}\right)+\gamma_{L S}+\gamma_{L V}+\varepsilon(t)
$$

At a given pressure $P_{V}$ the thickness $t$ of the adsorbed film is provided by minimizing $\Delta \Omega$ with respect to $t$. We write:

$$
\left(\frac{\partial \Delta \Omega}{\partial t}\right)_{P_{V}}=0 \Rightarrow \frac{d \varepsilon}{d t}=\rho_{L} k T \ln \left(\frac{P_{V}}{P_{0}}\right)
$$

Owing to conditions (4) that $\varepsilon$ has to meet, (9) can be integrated in the form:

$$
\varepsilon(t)=-\rho_{L} k T \int_{t}^{\infty} \ln \left(\frac{P_{V}}{P_{0}}\right) d \tau
$$

According to the Gibbs theory of adsorption, the system can also be described by the solid, the gas phase and an interface whose effective interface energy is $\gamma_{S V}^{\text {eff }}$. With such a description, equation (6) reduces to:

$$
\Delta \Omega / A=\gamma_{S V}^{e f f}
$$

Equating (8) and (11), one obtains:

$$
\gamma_{S V}^{e f f}(t)=-t \rho_{L} k T \ln \left(\frac{P_{V}}{P_{0}}\right)+\gamma_{L S}+\gamma_{L V}+\varepsilon(t)
$$

Relation (11) shows that $\gamma_{S V}^{e f f}=\gamma_{S V}$ as $t=0$. Taking into account (10), differentiation of (12) gives: 


$$
d \gamma_{S V}^{e f f}=-t \rho_{L} k T d \ln P_{V}
$$

Interestingly, noting $\rho_{L} t=\Gamma$ the amount of adsorbed liquid per surface area and $\mu_{V}$ the vapor specific chemical potential, from (13) we recover the celebrated Gibbs' adsorption equation, namely:

$$
d \gamma_{S V}^{e f f}=-\Gamma d \mu_{V}
$$

Using equation (10), the value of the coupling parameter $\varepsilon(t)$ can be calculated as a function of the experimental adsorbed layer thickness $t$. We fitted the coupling term using the experimental data reported by Kruk et al. ${ }^{36}$ for Ar adsorption at $87 \mathrm{~K}$ on a macroporous silica sample. As can be seen in Figure 2, experimental data are reasonably fitted using the exponential form given in equation (5). The data were also fitted by a $C / t^{2}$ law (not shown), which corresponds to the introduction of the Hamaker constant in the coupling term. Surprisingly, the exponential form of the coupling energy is much better than that generally used for van der Waals interactions in the disjoining pressure theory. ${ }^{34,37,38}$ There are several reasons explaining that the $C / t^{2}$ law might be a rather rough approximation. The first one may be that it only relies on $0 \mathrm{~K}$ adsorbate/surface potential energy arguments hence not taking into account any entropic contribution. The second may be related to the approximations in the used adsorbate/surface potential. The usual $C / t^{2}$ law is indeed derived from the two successive integrations starting from an oversimplified atom-atom attractive interaction potential form $\left[-\mathrm{Cte}^{6} \mathrm{r}^{6}\right]$ : the first one is carried out between one atom of the adsorbed film phase and all atoms in the solid slab located as a distance $z$ away (the integration is done on a semi-infinite space, the result is a $-c t e / z^{3}$ dependence of the energy); the second corresponds to the inclusion of other atoms in the adsorbed slab and gives the cte/ $z^{2}$ dependence. Nevertheless, the description of the atom-atom interaction in such an approach is very simplistic since it assumes that dispersion in the only source of (attractive) interaction. This is certainly not the case when considering the Ar/silica system since the partial charges onto the silica species create a non-zero permanent electric field close to the surface that gives rise to an attractive so-called polarization or induction interaction. Furthermore focussing on dispersion interaction only, it is know since the work of Barker, ${ }^{39}$ that restricting interaction to a two-body function only does not allow describing proper interfacial properties at the atomistic scale due to missing dispersion three-body interactions (that are not necessarily attractive, especially at interfaces). Finally, a complete interaction potential would also 
include a repulsive term that is often represented as an exponential form that decreases as distance increases so as in a Buckingham type of interatomic potential function. ${ }^{40}$ This repulsive (positive) term acts and dominates at short range (distance smaller than a few angstroms): it ensures the finite compressibility of matter and is related to the spatial extent of electronic wave functions. Thus one can infer that successive integrations of such a term will give to the final function an exponential behaviour. So the $C / t^{2}$ law rather corresponds to a first approximation of a more complex natural situation that is taken into account in experimental data enabling deriving a $\varepsilon(t)=\exp \left(-\frac{t}{\xi(T)}\right)$ expression. As can be seen in Figure $2, \xi(\mathrm{T})$ is on the order of a few angstroms.

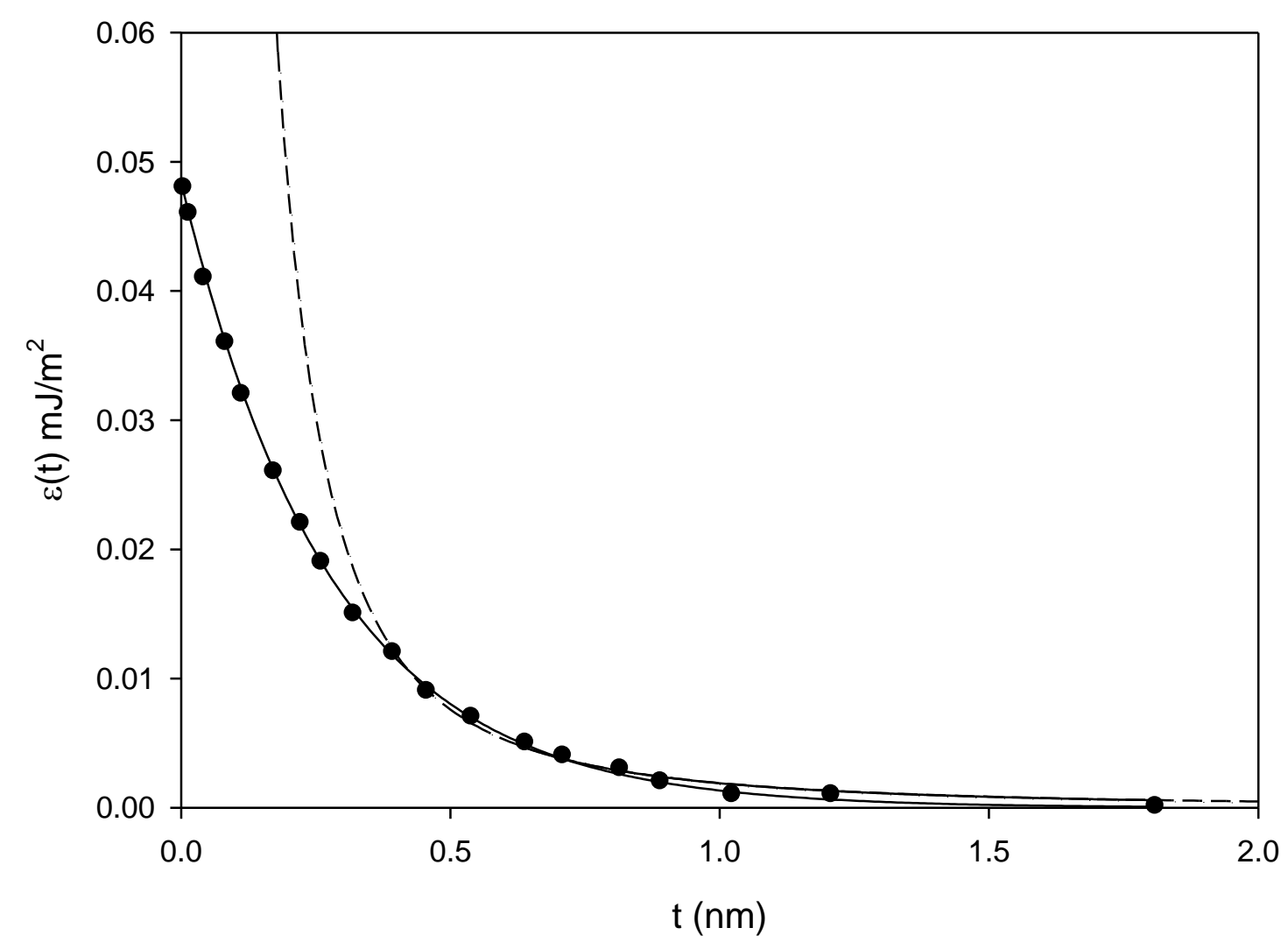

Figure 2. Evolution of the coupling parameter $\varepsilon(t)$ as a function of the adsorbed film thickness $t$ for argon at $87 \mathrm{~K}, \rho_{L}=1.394 \mathrm{~g} / \mathrm{cm}^{3}$, using $\varepsilon(t)=\exp \left(-\frac{t}{\xi(T)}\right)$ as a fitting function with $\xi(\mathrm{T})=0.278 \mathrm{~nm}\left(\right.$ solid line); using $\varepsilon(t)=A / t^{2}$ with $A=1.89610^{-3} \mathrm{~mJ} / \mathrm{m}^{2}$.

Twenty years ago Cheng and Cole ${ }^{41}$ derived a theoretical model for a thick film of simple adsorbate such as argon on some model surfaces such as graphite, silica, silicon, etc... within 
the frame of the Dzyaloshinskii, Lifshiz and Pitazvskii theory (DLP). In particular for thin films, they shown that the Frenkel-Halsey-Hill equation $\left(\mathrm{kT} \ln (\mathrm{P} / \mathrm{P} 0)=\mathrm{A}^{\prime} / \mathrm{t}^{-3}\right)$ that describes the interaction between adsorbed molecules with a plane substrate in the non retarded limit and from which on can recover the $\mathrm{A} / \mathrm{t}^{2}$ law after a further integration step, corresponds to "a difference between the energy of adding an atom to a film of thickness $t$ and the corresponding value for a hypothetical substrate constructed of the same material as the adsorbate; entropy and many-body terms are neglected". This is well on line with the above discussion on the validity of the $\mathrm{A} / \mathrm{t}^{2}$ law for describing the interaction between two solid bodies at short distances and missing three-body and higher order interactions that may contribute up to $\sim 20 \%$ of the film/substrate interaction energy.

\section{Application to capillary condensation in rigid cylindrical mesopores}

We now extend the previous model to gas-liquid transition of simple fluids in cylindrical mesopores having a finite length. Let us consider a cylindrical pore of radius $R_{0}$ having a length $L$. At low pressures, the pore surface is covered by a nanoscopic adsorbed film having a thickness $t=R_{0}-R$ (see Figure 3 ). The system is in equilibrium with the gas phase contained in the remaining of the pore volume, i.e. a cylindrical core of radius $R$. We note that this three phases system (adsorbent + adsorbed film + gas corresponding to phase 1,2 and 3 in Figure 1) is in equilibrium with an external bulk reservoir imposing its chemical potential and temperature so that the pressure is also imposed. The Gibbs free energy per unit of length is given by: ${ }^{32}$

$$
\frac{\Delta \Omega(R)}{\pi L}=-P_{V} R^{2}-P_{L}\left(R_{0}^{2}-R^{2}\right)+2 \gamma_{L S} R_{0}+2 \gamma_{L S} R+2 S R_{0}\left[\exp \left(\frac{R-R_{0}}{\xi(T)}\right)-\exp \left(\frac{-R_{0}}{\xi(T)}\right)\right]
$$

In addition, it is convenient to rewrite the change in grand free energy in equation (15) as a sum of a constant contribution and a contribution that depends on $R$, i.e. $\frac{\Delta \Omega(R)}{\pi L}=F_{0}+F(R)$ : 


$$
\begin{gathered}
F_{0}=-P_{L} R_{0}^{2}+2 \gamma_{L S} R_{0} \\
F(R)=-R^{2} \rho_{L} k T \ln \left(\frac{P_{V}}{P_{0}}\right)+2 \gamma_{L V} R+2 S R_{0} \exp \left(\frac{R-R_{0}}{\xi(T)}\right)
\end{gathered}
$$

In (17), since $R_{0}>>\xi(T)$ we neglected the last term in (16), while the Kelvin law (7) has been used.

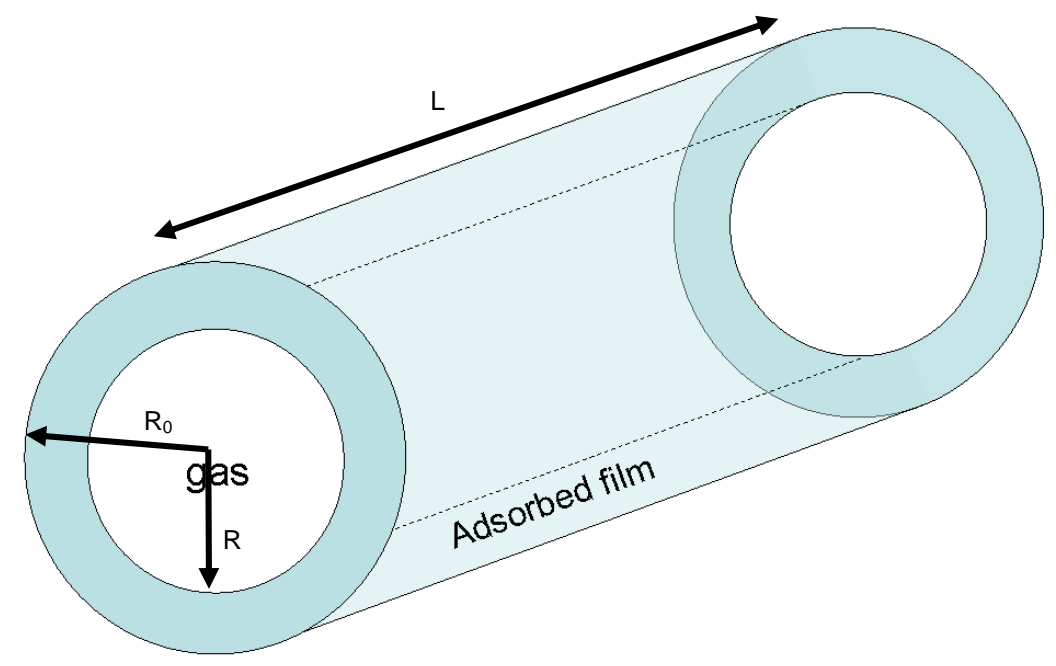

Figure 3. Schematic view of the cross section of a cylindrical pore of radius $R_{0}$ (adsorbent) showing the equilibrium between an adsorbed film at the pore surface and the gas phase in the pore center. The thickness of the adsorbed film is $t=R_{0}-R$.

$F(R)$ at constant temperature $T$ is shown in Figure 4 as a function of $R$ for different values of the gas pressure, $P_{V}$. The grand free energy exhibits two minima at low gas pressures. The stable solution $R \neq 0$ consists of a configuration where an adsorbed film at the pore surface is in equilibrium with the gas phase located in the pore centre. The metastable solution $R=0$ corresponds to the situation where the pore is completely filled with the liquid. As the pressure increases, the thickness of the adsorbed film increases and the minimum $R \neq 0$ is shifted toward the pore centre $R=0$. The equality of the two minima defines the pressure, $P_{\mathrm{e}}$, where capillary condensation should occur, provided that such a first order phase transition is 
an at equilibrium process. At this particular pressure, the system should exhibit phase coexistence between parts of the pore that are completely filled and parts where the system is composed of an adsorbed film in equilibrium with the gas. From a mathematical point of view, $P_{\mathrm{e}}$ must verify the conditions $\Omega(R)=\Omega(0)$ and $\frac{\mathrm{d} \Omega(R)}{d R}=0$. After a little algebra, it can be shown that these two criteria imply: ${ }^{32,42}$

$$
\ln \left(\frac{P_{e}}{P_{0}}\right)=-\frac{2 \gamma_{L V}}{k T \rho_{L} R}\left[1+\frac{2 \xi}{R-2 \xi}\right]
$$

As previously noted by Celestini, ${ }^{32}$ equation (18) is a generalized version of the modified Kelvin-Laplace equation, ${ }^{9,10}$ corrected for curvature effect due to the finite size of the pore radius. Given that $\xi$ is of the order of a few $\AA$ (see above), equation(18) reduces to the Kelvin equation for large pores $\xi<<R_{0}$, i.e. when $\frac{2 \xi}{R-2 \xi} \sim 0$. This generalized Kelvin equation can be considered as an alternative route to the modified Kelvin equation used in standard pore characterization methods, which include ad hoc corrections for the presence of an adsorbed film..$^{9,10,43,44,45,46,47}$ In contrast, the present model includes de facto the adsorbed film and allows us to predict the dependence on the gas pressure of its thickness, $t=R_{0}-R$. It can be used to calculate entire adsorption/desorption isotherm (including condensation and evaporation phenomena) as shown below. The curvature term in equation (15) gathered with the $\gamma_{L V}$ surface tension is formally equivalent to the well-know Tolman expression of surface tension for curved interfaces. The physical origin of this curvature correction is a difference between the distance of the equimolar surface and the surface of tension. As it is related to a curved interface, the $\gamma_{L V}$ surface tension should also obey the Tolman expression. As a result, Equation (17) can be rewritten: 


$$
\gamma_{L V}(R)=\gamma_{L V}(R \rightarrow \infty)\left[1+\frac{2 \xi}{R-2 \xi}+\theta\left(\frac{1}{R}\right)^{2}\right]
$$

The last equation is again formally close to the Tolman equation, ${ }^{48,49,50}$ originally derived for finite size objects such as clusters or droplets. $\xi$ can be then considered as being equal or proportional to the Tolman length with the same temperature dependence. ${ }^{51}$ Furthermore, we note that the plus sign in equations (18) and (19) is the consequence of the problem symmetry, i.e. a gas phase surrounded by a liquid layer. This can be discussed in term of the sign of the Tolman length itself. A positive value for the Tolman length corresponds to a situation in which the interface tends to curve toward the liquid phase, whereas a negative Tolman length implies a preferred curvature toward the vapor phase. The actual critical behaviour of the Tolman length was recently addressed by Anisimov who showed that it depends on the level of theory and that the amplitude of its divergence at a critical point is related to the degree of asymmetry in the fluid phase coexistence. ${ }^{52}$ In the case of a van der Waals fluid in a droplet, the Tolman length is finite (and negative) at the bulk critical point temperature $[\delta=0.2 \sigma]{ }^{53}$ Coming back to the analysis of free energy curves in Figure 4, $F(R)$ still exhibits two minima for pressures above $P_{\mathrm{e}}$ but the deepest solution is located at $R=0$, which corresponds to the pore completely filled with the liquid. The minimum $R=R_{\mathrm{K}}$ (adsorbed phase + gas) now corresponds to a metastable state. Condensation within the pore occurs as the pressure reaches $P_{\mathrm{c}}$ that corresponds to a limit of metastability; the condition of spontaneous liquid nucleation thus obeys the condition $\frac{\mathrm{d} \Omega(R)}{d R}=\frac{\mathrm{d}^{2} \Omega(R)}{d R^{2}}=0$, which implies that: $:^{32,42}$

$$
\ln \left(\frac{P_{c}}{P_{0}}\right)=-\frac{\gamma_{L V}}{k T \rho_{L} R}\left[1+\frac{\xi}{R-\xi}\right]
$$




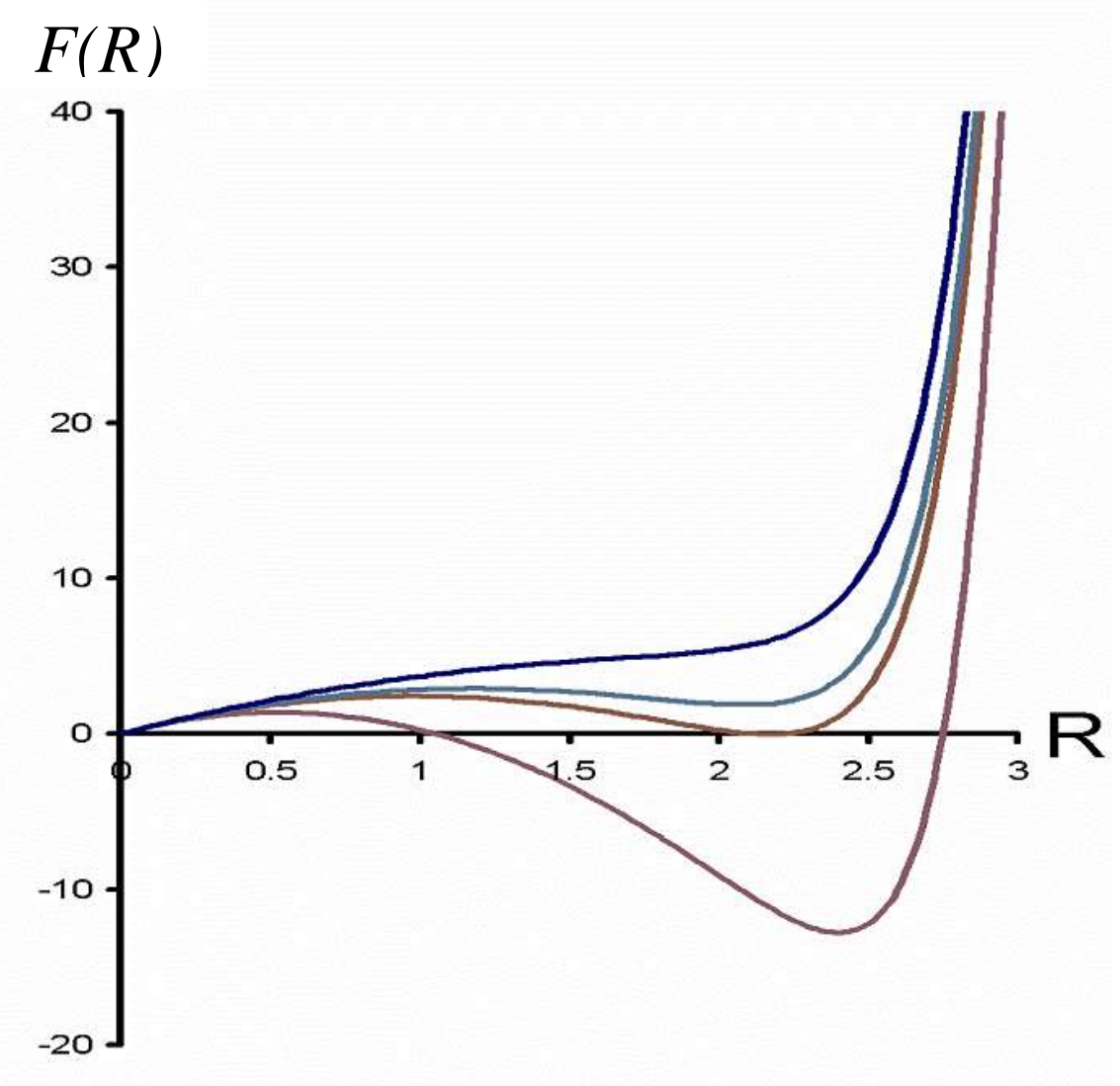

Figure 4. $F(R)$ at constant temperature $T$ for increasing pressures of the gas phase, $P_{V}$ (from bottom to top). The core radius $R$ is in $\mathrm{nm}$, the grand free energy $\Omega$ is in $10^{-19} \mathrm{~J}$ (see text).

For an infinitely long pore, evaporation can only occur through a cavitation process i.e. nucleation of a gas bubble within the cylindrical pore. ${ }^{54,55,56,57,58,59}$ It can be shown with similar calculations to those described above that cavitation takes place at a pressure close to the limit of stability of the metastable liquid phase (spinodal limit). Such a nucleation process occurs at very low pressure, $P_{\mathrm{d}}$, leading to a very large hysteresis loop.

For real porous materials made up of regular cylindrical pores having a finite length (MCM41, SBA-15), cavitation is not a relevant evaporation mechanism since the confined liquid phase is at all pressures in equilibrium with the external gas phase through the pore opening. As a result, the gas + adsorbed phase system is spontaneously nucleated within the pore when the pressure reaches the equilibrium transition pressure $P_{\mathrm{e}}$. Previous models of capillary condensation also assumed that capillary evaporation occurs at the equilibrium pressure. ${ }^{20,60,61}$ 
In the present model, capillary condensation occurs at the pressure $P_{\mathrm{c}}$, i.e. corresponds to a transition from the metastable gas + adsorbed phase to the stable liquid phase. We know from previous gas lattice calculations ${ }^{64}$ and molecular simulations ${ }^{62,65}$ that such a metastable capillary condensation for pores having a finite length (open at both ends) is close to that predicted for infinitely long pores. One can see that the capillary condensation pressure, $P_{\mathrm{c}}$, is larger than the equilibrium pressure, $P_{\mathrm{e}}$. This implies that the adsorption/desorption curve is irreversible, in agreement with experimental, theoretical, and simulation studies for unconnected mesopores, which have shown that the adsorption/desorption isotherms exhibit a hysteresis loop that should shrink as temperature increases and disappear at a so-called $T_{c c}$ temperature. $^{1}$

To summarize, in the present model, two different gas/liquid transition pressures are predicted; metastable condensation $P_{\mathrm{c}}$ and at equilibrium transition $P_{\mathrm{e}}$, which corresponds to an equilibrium evaporation process for a finite length pore. The (adsorbed film+gas) / liquid transition of the fluid within the pore is described as a first-order transition (involving phase coexistence and metastable states) in which the capillary condensation hysteresis is a van der Waals loop of the confined fluid. In addition, there is a critical temperature, $T_{c c}$, that corresponds to the temperature at which the hysteresis loop disappears in a mean field van der waals picture. We note that density functional theory (DFT) ${ }^{16,63,64,65}$ and van der Waals theories ${ }^{66,67}$ of confined fluids (mean field theories) also describe capillary condensation as a first order transition. The present model allows very fast estimates of the transition pressures that are in good agreement with experimental adsorption/desorption isotherms (see below). Interestingly, the present model, which includes de facto the existence of the adsorbed film, can be seen as a generalization of Cohan's theory for adsorption/condensation in pores open at both ends. ${ }^{20}$ 


\section{Results and Discussion}

Parameterization of the model: The parameters involved in this description of the capillary phenomenon in cylindrical pore fall into two categories: the first is concerned with physical parameters that can be obtained from other pieces of experiments; the second set contains the disposable parameters that have to be fitted against adsorption/desorption experiments. In a previous section, we have demonstrated that the choice of an exponential form for the coupling term in the Gibbs free energy can be derived from experimental data for adsorption on macroporous systems.

As shown below, we used $\xi(\mathrm{T})$ as an adjustable parameter for a whole series of adsorption/desorption data for a same MCM-41 materials at different temperatures in order to determine its dependence with temperature. Among other physical parameters, surface tensions of the vapor/adsorbent interface, $\gamma_{S V}$, and of the adsorbate/adsorbent interface, $\gamma_{L S}$, can be estimated from microcalorimetry measurements of the isosteric differential enthalpy of adsorption $\mathrm{Q}_{\mathrm{st}}$ at low coverage and at the capillary condensation pressure, respectively. This is done by converting the isosteric heat in energy per mole into an energy per unit of surface area using tabulated values for molecular cross-sections of the adsorbate $\left(a=\pi[\sigma / 2]^{2}=0.0908\right.$ $\mathrm{nm}^{2}$ for $\mathrm{Ar}$ and $0.1075 \mathrm{~nm}^{2}$ for $\mathrm{N}_{2} ; \sigma$ is the molecular kinetic diameter $\sigma=3.4 \AA$ for $\mathrm{Ar}$ and $\sigma=3.7 \AA$ for $\left.\mathrm{N}_{2}\right)$. For silica-based MCM-41 materials ${ }^{10}$, we found $\gamma_{\mathrm{SV}}=0.217 \mathrm{~J} / \mathrm{m}^{2}\left(\mathrm{Q}_{\mathrm{st}} \sim 15\right.$ $\mathrm{kJ} / \mathrm{mol})$ and $\gamma_{\mathrm{SL}}=0.100 \mathrm{~J} / \mathrm{m}^{2}\left(\mathrm{Q}_{\mathrm{st}} \sim 6.5 \mathrm{~kJ} / \mathrm{mol}\right)$ for $\mathrm{N}_{2}$ and $\gamma_{S V}=0.238 \mathrm{~J} / \mathrm{m}^{2}\left(\mathrm{Q}_{\mathrm{st}} \sim 13 \mathrm{~kJ} / \mathrm{mol}\right)$ and $\gamma_{S L}=0.102 \mathrm{~J} / \mathrm{m}^{2}\left(\mathrm{Q}_{\mathrm{st}} \sim 7.0 \mathrm{~kJ} / \mathrm{mol}\right)$ for Ar. Note that the present work reports some comparison between the model and experiment in the case of confined argon only. It is shown that the model allows a quantitative description of both adsorption and capillary phenomenon. Similar quantitative results were also obtained in the case of nitrogen but are not reported here for the sake of shortness and clarity. 
It is clear that surface tensions are related to free energy hence there is not only an enthalpy contribution but also an entropic term in them. Here we choose to consider these entropy contributions in a single fudge parameter $\gamma_{\text {Seffective }}$ (that contains mainly the entropy gasliquid entropy difference). $\mathrm{Q}_{\mathrm{st}}$ being positive $\left(\mathrm{Q}_{\mathrm{st}}=-\Delta \mathrm{H}_{\mathrm{ads}}\right), \gamma_{\text {Seffective }}$ has to be negative. In total, the spreading parameter S (see equation 3) writes

$$
S=\frac{Q_{s t}(0)-Q_{s t}(\text { cond })}{a}+\gamma_{\text {Seffective }}-\gamma_{L V}
$$

Concerning the temperature behaviour of the liquid-gas surface tension (the liquid being here the adsorbed film on the pore cylindrical wall), one can assume that it writes:

$$
\gamma_{L V}(T)=\gamma_{L V}(T=0)\left[1-\frac{T}{T_{c c}}\right]^{\mu}
$$

The idea is that above $T_{c c}$, there is no more a (clear) adsorbate film/gas interface but a inhomogeneous confined fluid. With this description, $\gamma_{L V}$ becomes zero at $T=T_{c c}$ giving $T_{c c}$ the status of a confined critical temperature associated with the first order phase [adsorbate film+ gas] to [condensed fluid] transition with the existence of an adsorbate-film/condensed liquid phase coexistence as shown in Figure 4. As $R_{0}$ tends to infinity, $T_{c c}$ tends to $T_{c}^{3 D}$ hence the critical exponent $\mu$ associated with the gas-liquid surface tension should recover the universal value of 1.26. $\gamma_{L V}(T=0)$ is taken as the gas-liquid surface tension at zero temperature as calculated from atomistic approaches $\left(\gamma_{L V}(T=0)=0.0374 \mathrm{~J} / \mathrm{m}^{2}\right.$ for $\operatorname{Ar}$ and $\gamma_{L V}(T=0)=0.0293$ $\mathrm{J} / \mathrm{m}^{2}$ for $\mathrm{N}_{2}{ }^{68}$ ). In the model presented in this paper, temperature and curvature dependence of the gas-liquid surface tension are treated as independent variable, hence written as the product of two distinct terms. Another consequence of equation (22) is to make the model inoperative at $T=T_{c c}$ exactly since the gas-liquid surface tension becomes zero. It is certainly the simplest way to produce a closing hysteresis loop model with increasing temperature 
conforming to the experimental temperature behaviour that is given by the relation $T_{c c}=\left[1-2 \sigma / R_{0}\right] T_{c}^{3 D} \cdot 11,21,69,70,71$

To summarize, the three disposable parameters of the present model are 1) the $\mu$ exponent in the definition of $\gamma_{L V}$ that is only pore-size dependent, 2) the entropy component of $\gamma_{S V}$ and $\gamma_{S L}$,

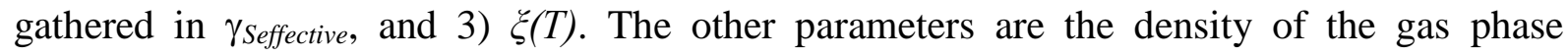
$\rho_{g}$ taken as that of a ideal gas and that of the (bulk) liquid or the adsorbed film, $\rho_{L}$, assumed to be that of the corresponding bulk Lennard-Jones fluid. Note that molecular simulation of capillary condensates have shown that their density is close to that of the bulk liquid. ${ }^{72}$ The saturating vapour pressure was calculated using the Kofke equation of state ${ }^{73}$ using both the energy and size Lennard-Jones potential parameters $(\varepsilon=120 \mathrm{~K}$ and $\sigma=3.4 \AA$ in the case of argon, $\varepsilon=94 \mathrm{~K}$ and $\sigma=3.7 \AA$ for nitrogen). We also considered the bulk critical temperature of the model fluid as defined by the Lennard-Jones energy parameter $\left(T_{c}^{3 D}=1.31 \varepsilon\right)$. As a consequence, the temperature values in the various calculations, were considered with respect to this value while experimental data were located in temperature with respect to the experimental $T_{c}^{3 D}$ value $\left(150.7 \mathrm{~K}\right.$ for $\mathrm{Ar}$ and $126.2 \mathrm{~K}$ for $\left.\mathrm{N}_{2}\right)$.

Calculation of argon condensation/evaporation isotherms: By fitting against one hysteretic adsorption isotherm, one can obtain the three adjustable parameters $\left(\gamma_{\text {Seffective }}, \mu\right.$ and $\xi)$ for a given fluid and pore radius at one temperature. The adsorption isotherm is calculated from a set of $F(R)$ versus $R$ curves (similar to those presented in Figure 4): the adsorbate film thickness is transformed into an adsorbed quantity (for instance $\mathrm{cm}^{3} \mathrm{STP}$ per gram of porous material) by assuming that it has the same density as that of the bulk liquid at the same temperature and by considering the specific surface area $\left(\mathrm{m}^{2} / \mathrm{g}\right)$ as a scale parameter which in turn can be transformed into a specific length $(\mathrm{m} / \mathrm{g})$ knowing the pore radius (note that the 
experimental specific length in the case of MCM type of mesoporous silica materials, is on the order of $10^{+19} \mathrm{~m} / \mathrm{g}$ !). In fine, a single fit of the adsorption branch of one adsorption/desorption isotherm allows the determination of the specific surface area with certainly a better accuracy than the standard BET approach for specific surface areas determination. Here we make a clear difference between the BET model $l^{68}$ that is an interesting simple model for multilayer adsorption on a plane surface and the BET method for assessing specific surface $\operatorname{area}^{10}$ that, in essence, ignores the main assumption of the BET model stating that adsorbed molecules in different adsorption sites do not interact (even angstromly close); the only adsorbate-adsorbate interaction that is retained being the energy of molecules adsorbed on the top of each other in the same adsorption site. In the BET method, one considers the surface as covered with a dense packing of (hence interacting) adsorbed molecules forming the adsorbed film. In the present work, the existence of a growing dense (liquid-like) adsorbed film is naturally described. With the model presented in this paper, one also predicts hysteretic isotherms with vertical jumps at condensation and (equilibrium) evaporation in agreement with other theoretical (DFT) or simulation studies of a simple fluid confined in cylindrical mesopores. Recent simulation and theoretical results in disordered and constricted mesopores have shown that these extended defects are responsible for the nonverticality of both adsorption/desorption branches in condensation/evaporation isotherms. ${ }^{24,25,26,55,56}$ We shall demonstrate that with the present model, the equilibrium evaporation branch is naturally located close to the pressure corresponding to hysteresis loop closure point. We tested the model by comparing its performances first with experimental data for argon measured at constant pore size but with varying temperature and second with constant temperature experimental results measured for a variety of MCM pore sizes.

Figure 5 presents some results for argon confined in a $4.2 \mathrm{~nm}$ pore (in diameter) as a function of temperature. The various adsorption/desorption isotherms were obtained with the fixed 
values $\mu=0.4$ (adjusted on the first case then kept constant) and $\gamma_{L V}=-0.075 \mathrm{~J} / \mathrm{m}^{2}$ and $\xi$ as the only adjustable parameter. One can see that the agreement with experiment ${ }^{74}$ is rather good for such a simple (nearly analytical) model. Note $(i)$ that the model allows a very good description of the adsorbed film at all temperatures except when very close to $T_{c c}$ and (ii) that the temperature description of the hysteresis width is good up $93 \%$ of the value of $T_{c c}$ thus showing good performances for temperatures not too close to this critical value. It is not surprising since the model presented here is in essence a mean field approach that does not allow considering thermally-driven fluid density fluctuations. As expected, the model indicates that desorption in finite length pores occurs close to equilibrium. Indeed the desorption line in the theoretical curves corresponds to the equilibrium pressure as given by the generalized Kelvin equation. Interestingly, this simple model for capillary condensation does not require a geometrical description of interfacial menisci as originally proposed in the Cohan approach ${ }^{20}$ that explains the hysteresis loop in adsorption/desorption isotherms as a consequence of the change in the symmetry of the gas/liquid interface from cylindrical to hemispherical shape. Finally, Figure 5 shows that the relative height of the capillary jump with respect to the adsorbed amount at the onset of condensation is well reproduced, which shows the ability of the model in estimating the specific surface area. The model presented here does not give an account of the confined liquid compressibility as shown in Figure 5. Interestingly, $\xi$ mainly acts on the lower part of the adsorption/desorption isotherm prior to capillary phenomena allowing a good fitting of experimental data (see Figure 5). Figure 6 gives the evolution of the $\xi$ parameter as a function of $\left[t=1-T / T_{c}^{3 D}\right] . \xi$ linearly decreases with temperature in a way very similar to that found for nanoclusters within the van der Wall mean field model. ${ }^{53}$ By extrapolating $\xi$ at $t=\left(1-T / T_{c}\right)=0$, one recovers a value of $\xi$ close to $0.2 \sigma$. These arguments are in favour for identifyng $\xi$ to the Tolman length. As mentioned above, the present model is in essence a mean field approach. Therefore, one expects to find mean-field 
results compatible with those established in the context of density functional theories for a liquid droplet coexisting with a gas phase. These theories give consistent results with regard to the mean-field value of the Tolman length for simple liquids: it is only weakly temperature dependent reaching a value at the critical point which is a small fraction of the molecule diameter and negative, and is given by the difference of the correlation lengths of two bulk phases. Tolman himself showed that the Tolman length can be expressed in terms of the adsorbed amount at the surface of tension at coexistence. ${ }^{48,49}$ In our geometry (gas phase surrounded by a layer of liquid as a consequence of the adsorption process), it is not surprising to find a positive Tolman length. Working at constant pore size, the calculated data in Figure 5 were obtained for constant value of the $\mu$ exponent (see equation 21). Figure 7 presents calculated adsorption/desorption isotherms for $\mathrm{Ar}$ at $87 \mathrm{~K}$ confined in MCM-41 pores of various sizes. Since these are constant temperature calculations $\left(\mathrm{T}_{\text {cal }}=91.2 \mathrm{~K}\right)$, they were carried out for a constant value $\xi=2.2 \AA$. This value is not that expected from the linear relationship given in Figure 6 for the same temperature (from Figure 6, one expects $\xi=2.6 \AA$ ). Since $\xi$ has the status of the Tolman length and therefore is a direct consequence of the adsorbed film thickness, one can conceive that it depends on the system thermodynamics, particularly its adsorbate-adsorbent contribution. As a result, $\xi$ must depend on the chemical nature of the matrix but also on its density. As a consequence, one has to consider the Tolman length as being dependent of the adsorbent porosity. We propose to correct $\xi$ for fluidadsorbent interaction by multiplying it by the amount of solid in the porous material and given by (1- $\phi), \phi$ being the porosity. As already mentioned, data reported in Figure 6 were obtained for a cylindrical pore of radius $2.2 \mathrm{~nm}$; the corresponding $\xi$ parameter was estimated equal to 2.6 A. From Figure 7b, experimental data for a pore with radius equal $2.1 \mathrm{~nm}$ were best fitted with a $\xi$ parameter equal to $2.2 \AA$. These two porous materials have cylindrical pores of (almost) the same diameter but have different porosity $\phi_{1}$ and $\phi_{2}$. From a simple algebra, 

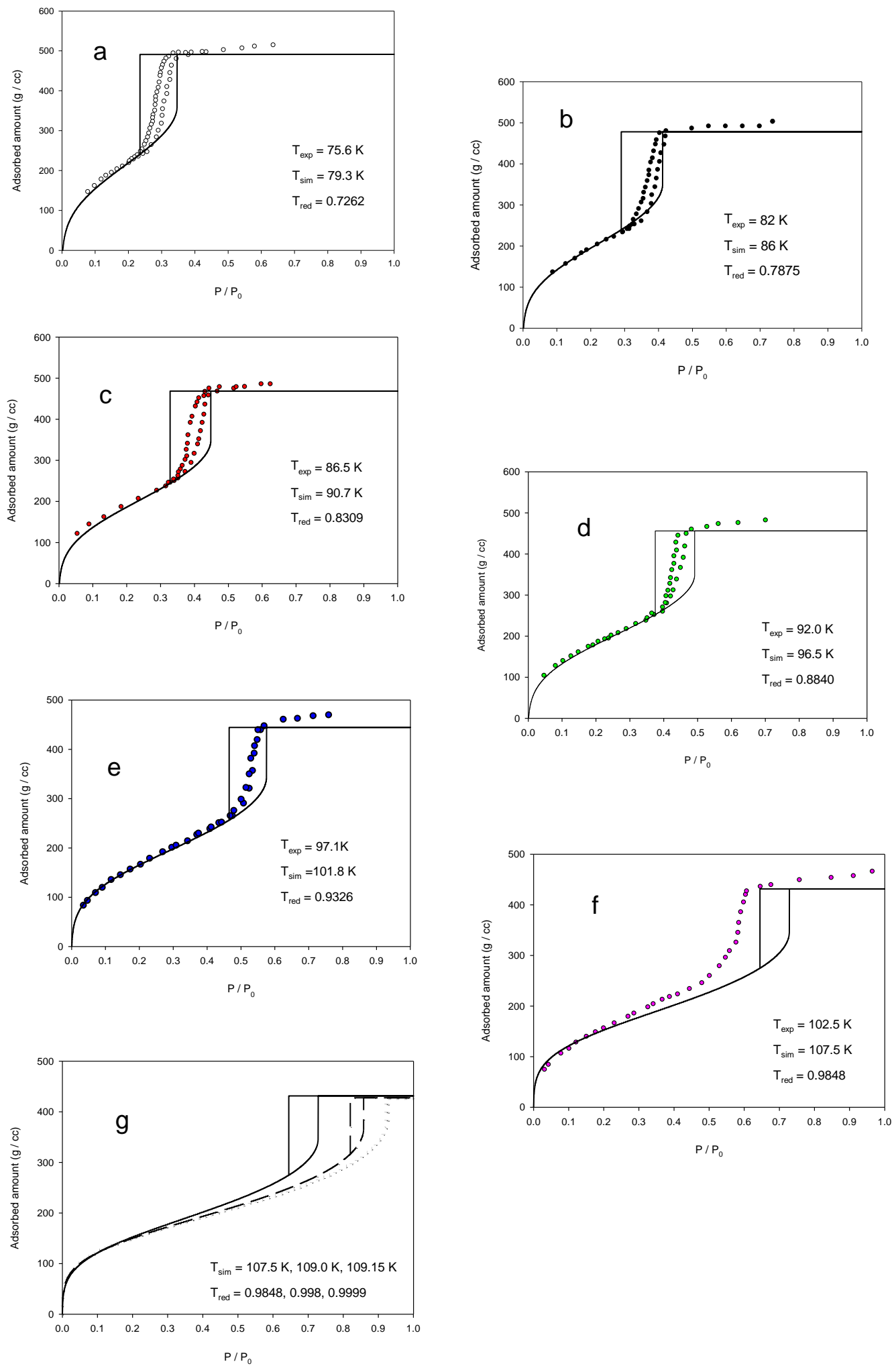

Figure 5: Ar adsorption isotherm in a $4.2 \mathrm{~nm}$ diameter pore at various temperatures. (a-f) comparison with experiment (points), model (lines), (g) calculated isotherms as the temperature approaches $T_{c c}$. Experiment and model are plotted at equal $\mathrm{T} / \mathrm{T}_{\mathrm{cc}}$ ratio. 
one can show that:

$$
\frac{\left(1-\phi_{1}\right)}{\left(1-\phi_{2}\right)}=\frac{V_{s p}^{2}}{V_{s p}^{1}}=\frac{S_{s p}^{2}}{S_{s p}^{1}} \approx 0.85
$$

where $V_{s p}{ }^{i}$ and $S_{s p}{ }^{i}$ are the specific volume and surface area of material $i\left(V_{s p}^{2}=460 \mathrm{~cm}^{3}\right.$ $\mathrm{STP} / \mathrm{g}, V_{s p}^{1}=540 \mathrm{~cm}^{3} \mathrm{STP} / \mathrm{g}$, and $\left.S_{s p}^{2}=550 \mathrm{~m}^{2} / \mathrm{g}, \quad S_{s p}^{1}=650 \mathrm{~m}^{2} / \mathrm{g}\right)$. The values for specific surface area are given by the model while specific volume data can be directly obtained from experimental adsorption/desorption isotherms at pressures larger than that corresponding to the capillary pressure, assuming that the confined capillary liquid has the same density as in the bulk. This latter assumption is supported by atomistic simulations of confined simple fluid in mesopores. ${ }^{75}$ Assuming that the two materials have the same pore size (within $1 \AA$ ), we propose a porosity independent $\xi$ parameter that can be obtained following the relationship:

$$
(1-\phi) \xi=\xi_{\text {material }}
$$

Where the porosity dependent $\xi_{\text {material }}$ parameter is the one obtained by fitting against the experimental data.

Thus comparing the two materials, at the same temperature, one gets

$$
\frac{\left(1-\phi_{1}\right)}{\left(1-\phi_{2}\right)}=\frac{\xi_{\text {material }}^{1}}{\xi_{\text {material }}^{2}}=\frac{2.2}{2.6} \approx 0.85
$$

in good agreement with the value obtained from geometric considerations (see equation 23). For a given porous material, there is a simple relation between its specific pore volume and its density; $1 / V_{s p}=\rho_{\text {wall }}(1-\Phi)$ where $\rho_{\text {wall }}$ is equal to $2.2 \mathrm{~g} / \mathrm{cm}^{3}$ for silica.

One can see from Figure 7 that the model predicts very well the adsorbed film for all the considered pore sizes. The hysteresis loop is also well described as long as the pore size is not too small for the same reasons as for the temperature study at constant pore size: decreasing the pore size at constant temperature is a way to make the fixed temperature matching $T_{c c}$ 
hence reaching the model capability. Figure 8 gives the evolution of the $\mu$ exponent as a function of pore size. As a validation of the present approach, $\mu$ does increase as the pore size increases and recovers the known value of the critical exponent of the surface tension for the

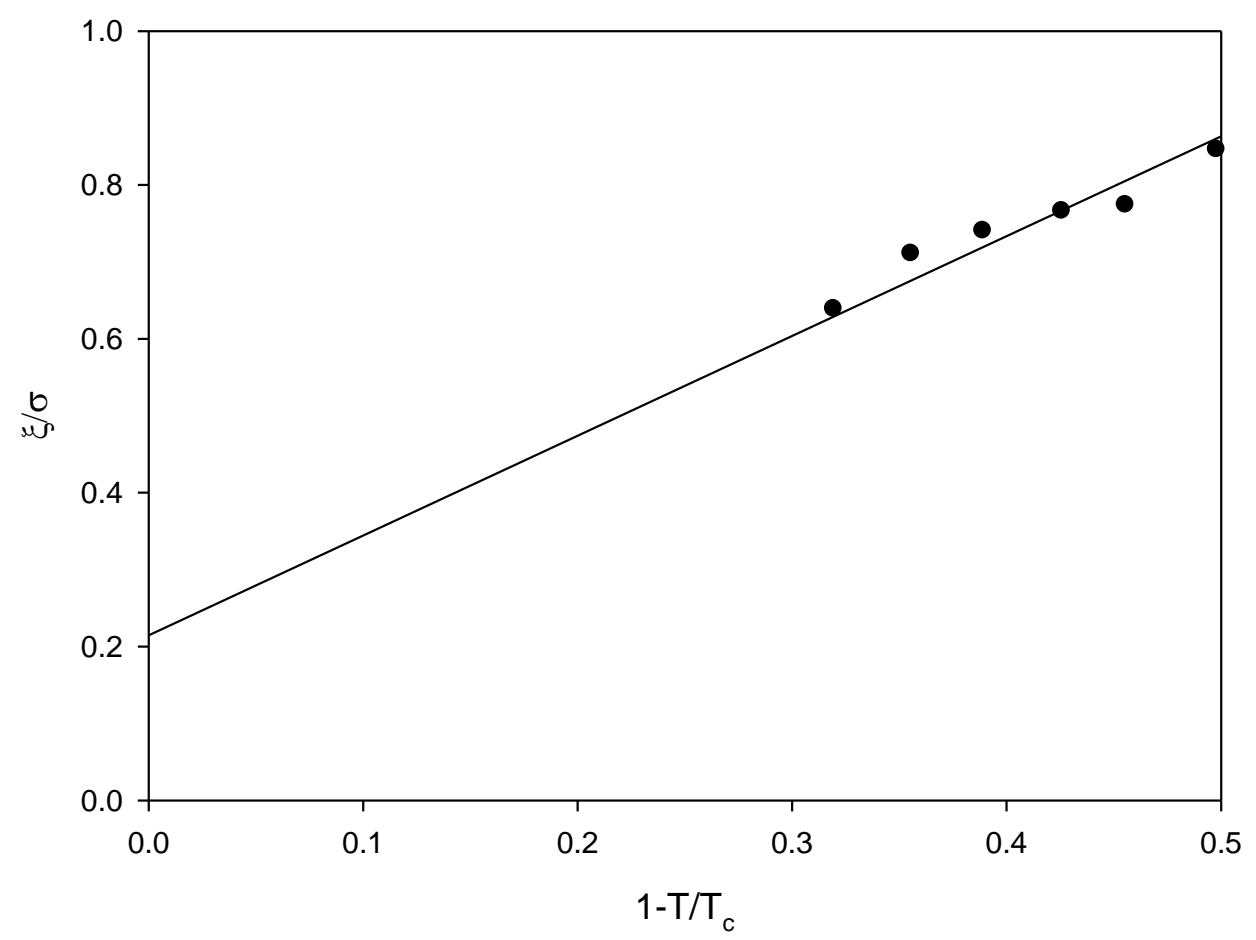

Figure 6: (Circles): Evolution of $\xi$ (reduced by the molecular kinetic diameter) as a function of the reduced temperature (with respect to $T_{c}$ ). The line is a linear regression.

flat interface $(\mu=1.26)$. We have characterized the evolution of the main model parameters, namely $\xi$ and $\mu$, with their corresponding variables $T$ and $R_{0}$ respectively. Interestingly the entropy contribution to the spreading parameter $S$ (see Equation 21) is found again constant at $\gamma_{\text {Seffective }}=-0.075 \mathrm{~J} / \mathrm{m}^{2}$ (similar calculation for nitrogen gives $\gamma_{\text {Seffective }}$ constant at about -0.035 $\mathrm{J} / \mathrm{m}^{2}$ ). This is also the case for enthalpic contributions to the spreading parameter $S$. This reflects the fact that the isosteric heat of adsorption variations with loading is very much insensitive to temperature as found in Grand Canonical Monte-Carlo simulation of adsorption of argon in many silica pores. ${ }^{76}$ 

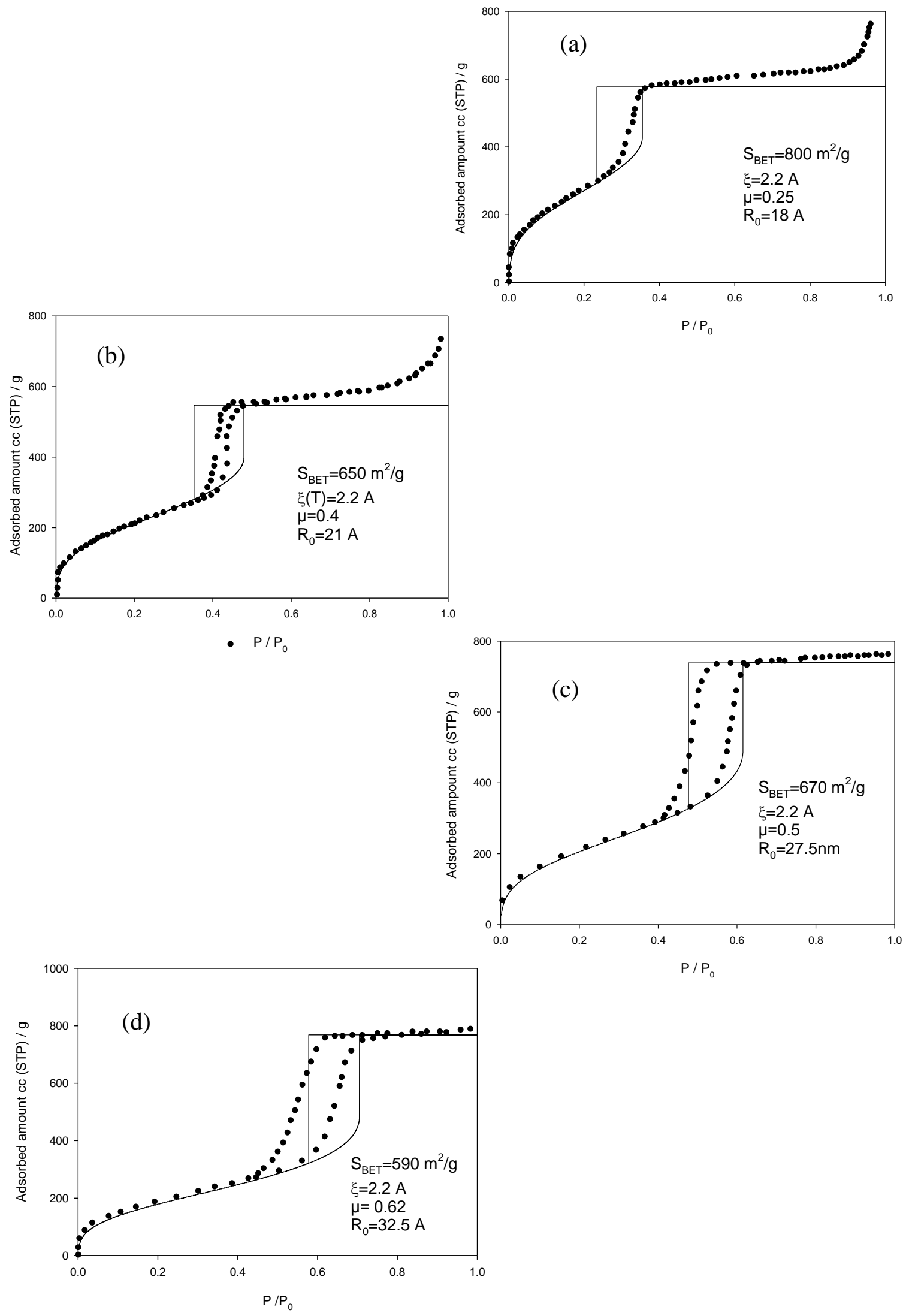
Figure 7: Ar adsorption at $87 \mathrm{~K}$ in various MCM type pores (a-d) comparison with experiment (points), model (lines)

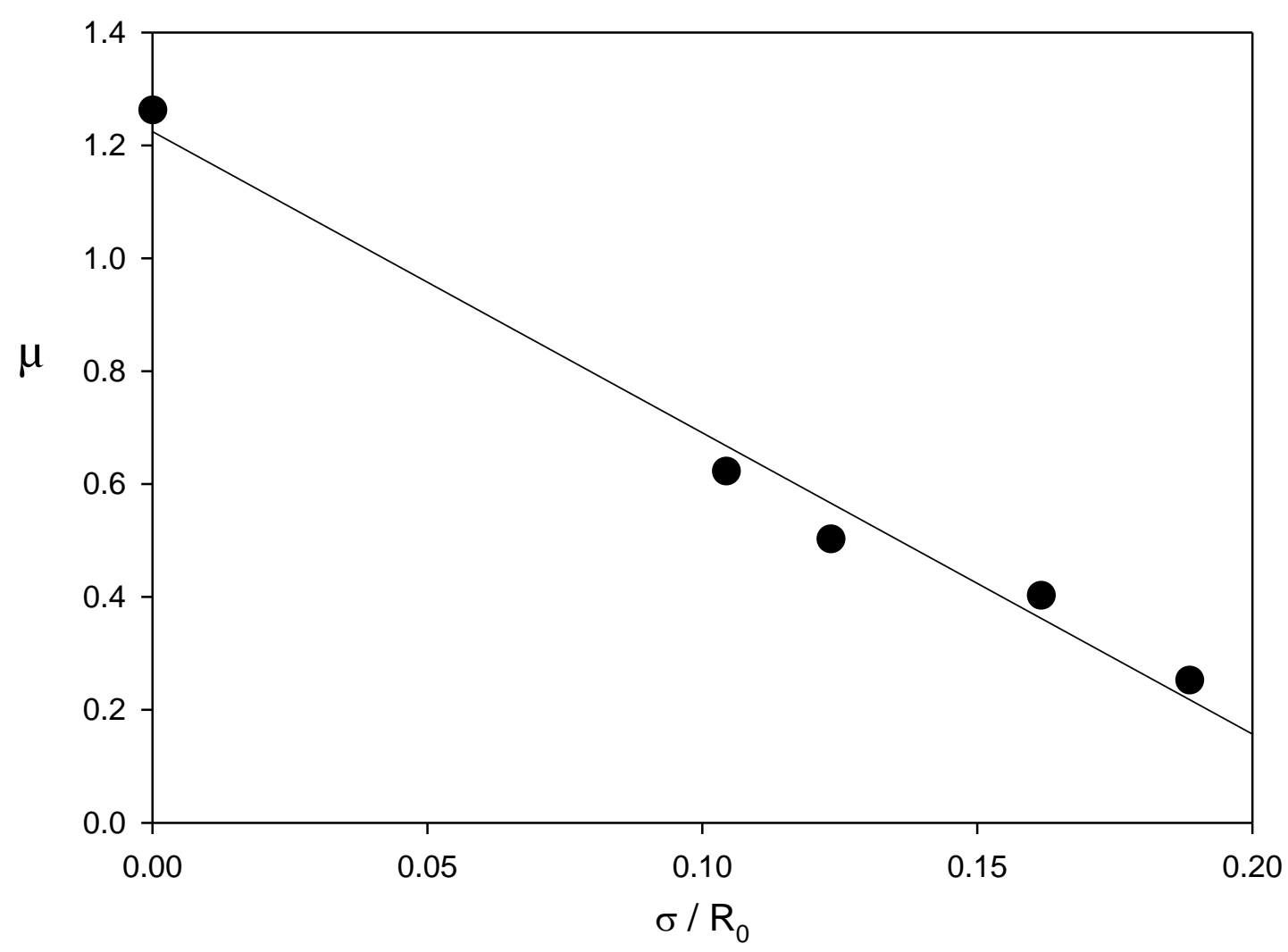

Figure 8: $\mu$ exponent in equation (22) as a function of the reciprocal of the pore size. The line is a linear regression.

\section{Conclusion}

In this paper, we have presented a mean field model for capillary condensation and evaporation of a molecular fluid confined in a finite cylindrical pore. This approach allows considering in a very compact way both surface adsorption and capillary condensation and evaporation transitions. We devoted a special attention to give to all used quantities and parameters a well defined physical meaning. Their behaviour as a function of temperature and confinement is presented. We have shown in the case of a plane surface that this approach, inspired by the work of Celestini (Phys. Lett. A, 1997, 228, 84), allows the Gibbs adsorption equation to be recovered. Furthermore in the case of a fluid confined to a finite length cylindrical pore, we have demonstrated that the Tolman length plays a central role in 
describing surface and confinement phenomena: a generalized Kelvin-Tolman equation could be derived that takes into account surface curvature effects. The model performances are checked against experimental data for Ar confined in MCM-41 materials of various pore sizes and at different temperatures by calculating full adsorption/desorption isotherms. Along an isotherm, it is shown that condensation occurs at the end of a metastable range of pressure while evaporation occurs at equilibrium. This model reasonably reproduces experimental results obtained for real solids such MCM-41 nanoporous silica provided that calculations are carried out at a temperature not too close to $T_{c c}$ defined as the pore critical temperature. This is due to the mean-field character of our approach. From a practical point of view, due to its simplicity and tractability, we have shown that our simple (nearly) analytical and integrated approach can be used for characterization purposes (giving realistic estimates of the specific surface area and mean pore size) from the fit of a single experimental adsorption/desorption isotherm curve. It can be viewed as an alternative to the BET, BJH/de Boer and FHH characterization tools of porous materials. 


\section{References}

${ }^{1}$ Gelb, L. D.; Gubbins, K. E.; Radhakrishnan, R.; Sliwinska-Bartkowiak, M. Rev. Prog. Phys. 1999, 62, 1573.

${ }^{2}$ Alba-Simionesco, C; Coasne, B.; Dosseh, G.; Dudziak, G.; Gubbins, K. E.; Radhakrishnan, R.; Sliwinska-Bartkowiak, M. J. Phys. Condens. Matter 2006, 18, R15.

${ }^{3}$ Bisi, O.; Ossicini, S.; Pavesi, L. Surf. Sci. Rep. 2000, 38, 1.

${ }^{4}$ Ajayan, P. M. in Carbon Nanotubes: Preparation and Properties ed. Ebbesen, T. W. 1996 (Chemical Rubber Company, Boca Raton, FL). Ajayan, P. M. Chem. Rev. 1999, 99, 1787.

${ }^{5}$ Beck, J. S.; Vartuli, J. C.; Roth, W. J.; Leonowicz, M. E.; Kresge, C. T.; Schmitt, K. D.; Chu, C. T.-W.; Olson, D. H.; Sheppard, E. W.; McCullen, S. B.; Higgins, J. B.; Schlenkert, J. L. J. Am. Chem. Soc. 1992, 114, 10834.

${ }^{6}$ Zhao, D.; Feng, J.; Huo, Q.; Melosh, N.; Fredrickson, G. H.; Chmelka, B. F.; Stucky, G. D. Science 1998, 279, 548.

${ }^{7}$ Levitz, P.; Ehret, G.; Sinha, S. K.; Drake, J. M. J. Chem. Phys. 1991, 95, 6151.

${ }^{8}$ Haller, W. in Solid Phase Biochemistry (ed. Scouten, W. H.) 1983 Chap. 11, 535 (Wiley, New York).

${ }^{9}$ Gregg, S. J.; Sing, K. S. W. in 'Adsorption, Surface area and Porosity 1982 (Academic press, London).

${ }^{10}$ Rouquerol, F.; Rouquerol, J.; Sing, K. S. W. in Adsorption by Powders and Porous Solids 1999 (Academic Press, London).

${ }^{11}$ Morishige, K.; Fujii, H.; Uga, M.; Kinukawa, D. Langmuir 1997, 13, 3494.

${ }^{12}$ Morishige, K.; Shikimi, M. J. Chem. Phys. 1998, 108, 7821.

${ }^{13}$ Morishige, K.; Ito, M. J. Chem. Phys. 2002, 117, 8036.

${ }^{14}$ Schreiber, A.; Reinhardt, S; Findenegg, G. H. Studies in Surface Science and Catalysis, 2002, Vol. 144 (Eds. Rodriguez-Reinoso, F., McEnaney, B., Rouquerol, J. and Unger, K. 
K.) 177 (Elsevier Science).

${ }^{15}$ Morishige, K.; Nakamura, Y. Langmuir 2004, 20, 4503.

${ }^{16}$ Ball, P. C.; Evans, R. Langmuir 1989, 5, 714.

${ }^{17}$ Everett, D. H. in The structure and properties of porous materials 1958, p. 95; Barker, J. A. ibid., p. 125.

${ }^{18}$ Mason, G. J. Colloid Interf. Sci.1982, 88, 36.

${ }^{19}$ Mason, G. Proc. Roy. Soc. Lond. 1988, 415, 453.

${ }^{20}$ Cohan, L. H. J. Am. Chem. Soc. 1938, 60, 433.

${ }^{21}$ Evans, R.; Marini Bettolo Marconi, U.; Tarazona, P. J. Chem. Phys. 1986, 84, 2376.

${ }^{22}$ Evans, R. J. Phys. Condens. Matter 1990, 2, 8989.

${ }^{23}$ Landau, L. D.; Lifshitz, E. M. in Statistical Physics 3rd Ed. 1959, Vol. 1 (Pergamon Press, Oxford).

${ }^{24}$ Kierlik, E.; Monson, P. A.; Rosinberg, M. L.; Sarkisov, L.; Tarjus, G. Phys. Rev. Lett. 2001, $87,055701$.

${ }^{25}$ Kierlik, E.; Rosinberg, M. L.; Tarjus, G.; Viot, P. Phys. Chem. Chem. Phys. 2001, 3, 1201.

${ }^{26}$ Kierlik, E., Monson, P. A., Rosinberg, M. L.; Tarjus, G. J. Phys.: Condens. Matter 2002, 14, 9295.

${ }^{27}$ Pellenq, R. J.-M.; Coasne, B., unpublished work

${ }^{28}$ Detcheverry, F.; Kierlik, E.; Rosinberg, M. L.; Tarjus G. Phys. Rev. E 2003, 68, 061504.

${ }^{29}$ Gibbs, J. W. Collected works of J. W. Gibbs 1928 (Longmans and Green, New York).

${ }^{30}$ Coussy, O.; Pellenq, R . J. -M.; Coasne, B.; Denoyel, R. O. to be submitted

${ }^{31}$ Denoyel, R. O.; Pellenq, R . J. -M. Langmuir 2002, 18, 2710.

${ }^{32}$ Celestini, F. Phys. Lett. A 1997, 228, 84.

${ }^{33}$ Celestini, F. Thesis 1995, University of Nice - Sophia Antipolis, France.

${ }^{34}$ De Gennes, P. G. Rev. Mod. Phys. 1985, 57, 827. 
${ }^{35}$ Evans, R.; Marini Bettolo Marconi, U.; Tarazona, P. J. Chem. Soc. Faraday Trans. 2 1986, $82,1763$.

${ }^{36}$ Kruk, M.; Jaroniec, M. Chem. Mater. 2000, 12, 222.

${ }^{37}$ Derjaguin, B. Acta Physicochim. URSS 1940, 12, 181.

${ }^{38}$ Neimark, A. V.; Ravikovitch, P. I.; Vishnyakov, A. J. Phys.: Condens. Matter 2003, 15, 347.

${ }^{39}$ Barker, J. A., Phys. Rev. Lett. 1986, 57, 230.

${ }^{40}$ Pellenq R. J.-M., Pellegatti A., Nicholson D., Minot C., J. Phys. Chem.,1995, 99, 10175.

${ }^{41}$ Cheng E, Cole M. W., Phys. Rev. B, 1988, 38, 987.

${ }^{42}$ Pellenq, R . J. -M.; Denoyel, R. O. in Fundamentals of Adsorption 7 2002, Eds. Kaneko, K.; Kanoh, H.; Hanzawa, Y., 352 (IK International Pub.).

${ }^{43}$ Kruk, M.; Antochshuk, V.; Jaroniec, M.; Sayari, A. J. Phys. Chem. B 1999, 103, 10670.

${ }^{44}$ Kruk, M.; Jaroniec, M.; Sakamoto, Y.; Terasaki, O.; Ryoo, R.; Ko, C. H. J. Phys. Chem. B 2000, 104, 292.

${ }^{45}$ Kruk, M.; Jaroniec, M. Chem. Mater. 2000, 12, 222.

${ }^{46}$ Kruk, M.; Jaroniec, M. J. Phys. Chem. B 2002, 106, 4732.

${ }^{47}$ Qiao, Z.; Bhatia, S. K.; Nicholson, D. Langmuir 20, 20, 389.

${ }^{48}$ Tolman, R. C. J. Chem. Phys. 1949, 17, 118.

${ }^{49}$ Tolman, R. C. J. Chem. Phys. 1949, 17, 333.

${ }^{50}$ Santiso, E.; Firoozabadi, A. AIChE J. 2006, 52, 311

${ }^{51}$ Blokhuis, E. M.; Kuipers, J. J. Chem. Phys., 2006, 124, 074701

${ }^{52}$ Anisimov, M. A.; Phys. Rev. Lett. 2007, 98035702.

${ }^{53}$ van Giessen, A. E.; Blokhuis E. M., Bukman D. J. J. Chem. Phys. 1998, 108, 1148.

${ }^{54}$ Sarkisov, L.; Monson, P. A. Langmuir 2001, 17, 7600.

${ }^{55}$ Coasne, B.; Pellenq, R. J. -M. J. Chem. Phys. 2004, 121, 3767. 
${ }^{56}$ Coasne, B.; Gubbins, K. E. ; R. J. -M. Part. Part. Syst. Charac. 2004, 21, 149.

${ }^{57}$ Ravikovitch, P. I.; Neimark, A. V. Langmuir 2002, 18, 1550.

${ }^{58}$ Ravikovitch, P. I.; Neimark, A. V. Langmuir 2002, 18, 9230.

${ }^{59}$ Vishnyakov, A.; Neimark, A. V. Langmuir 2003, 19, 3240.

${ }^{60}$ Cole, M. W.; Saam, W. F. Phys. Rev. Lett. 1974, 32, 985.

${ }^{61}$ Saam, W. F.; Cole, M. W. Phys. Rev. B 1975, 11, 1086.

${ }^{62}$ Maddox, M. W.; Gubbins, K. E. Langmuir 1995, 11, 3988.

${ }^{63}$ Ravikovitch, P. I.; Domhnaill, S. C. O.; Neimark, A. V., Schuth, F.; Unger, K. K. Langmuir 1995, 11,4765 .

${ }^{64}$ Marini Bettolo Marconi, U.; van Swol, F. Phys. Rev. A 1989, 39, 4109.

${ }^{65}$ Gelb, L. D. Mol. Phys. 2002, 100, 2049.

${ }^{66}$ Zarragoicoechea, G. J.; Kuz, V. A. Phys. Rev. E 2002, 65, 021110.

${ }^{67}$ Zarragoicoechea, G. J.; Kuz, V. A. Fluid Phase Equilibira 2004, $220,7$.

${ }^{68}$ Andamson, A. W. Physical chemistry of surfaces, Fifth Edition, John Wiley \& Sons, Inc., 1990.

${ }^{69}$ Pellenq R. J.-M., Coasne B., Denoyel R. O., and Puibasset J., 7th Int. Symp. on the Characterization of Porous Solids, in Studies in Surface Science and Catalysis, 160, 1, P.L. Llewellyn, F. Rodriguez Reinoso, J. Rouquerol and N. Seaton Eds. (2007).

${ }^{70}$ Coasne B., Gubbins K. E., Pellenq R. J.-M., Adsorption, 2005, 11, 289.

${ }^{71}$ Puibasset J., J. Chem. Phys., in press.

${ }^{72}$ Pellenq, R.J.-M., Levitz, P. Mol. Phys 2002, 100, 2059.

${ }^{73}$ Kofke, D. A. J. Chem. Phys. 1993, 105, 4149.

${ }^{74}$ Morishige, K.; Nakamura, Y Langmuir 2004, 20, 4503.

${ }^{75}$ R.J.-M. Pellenq, S. Rodts, V. Pasquier, A. Delville and P. Levitz, Adsorption, 2000, 6, 241.

${ }^{76}$ B. Coasne, R. J.-M. Pellenq, unpublished data. 